\title{
ON THE COMPOSITION OF COMPLETELY MONOTONIC FUNCTIONS AND COMPLETELY MONOTONIC SEQUENCES AND RELATED QUESTIONS
}

\author{
LEE LORCH AND DONALD J. NEWMAN
}

\section{Definitions}

A function $f(x)$, such as $e^{-x}$ or $(x-a)^{-\gamma}, \gamma \geqslant 0$, is said to be completely monotonic over $(a, b)$, where $-\infty \leqslant a<b \leqslant+\infty$, if

$$
(-1)^{n} f^{(n)}(x) \geqslant 0, \quad a<x<b, \quad n=0,1,2, \ldots
$$

If, in addition, $f(x)$ is continuous at $x=a$, then it is called completely monotonic over $[a, b)$, with similar definitions for $(a, b]$ and $[a, b]$. A function is absolutely monotonic if all its derivatives are non-negative. A detailed study of these concepts can be found, for example, in [11, Chapter IV].

Our chief concern here is for the standard case in which $a=0, b=+\infty$. For this, S. N. Bernstein established the following results (cf. [11, Theorems 12a and 12b, pp. $160-161]$ ).

THEOREM A. A necessary and sufficient condition that $f(x)$ be completely monotonic in $[0, \infty)$ is that there exist a bounded, non-decreasing function $\alpha(t)$ such that

$$
f(x)=\int_{0}^{\infty} e^{-x t} d \alpha(t)
$$

and that the integral converge for $0 \leqslant x<\infty$.

THEOREM B. For $f(x)$ to be completely monotonic over $(0, \infty)$ it is necessary and sufficient that $f(x)$ be representable in the form (2) with $\alpha(t)$ non-decreasing and the integral convergent for $0<x<\infty$.

J. Dubourdieu [4, p. 98] pointed out that strict inequality prevails in (1) for all non-constant functions completely monotonic over $(a, \infty)$; that is, if $f(x)$ satisfies (1) and is not constant, then

$$
(-1)^{n} f^{(n)}(x)>0, \quad a<x<\infty, \quad n=0,1,2, \ldots
$$

A completely monotonic sequence $\left\{\mu_{0}, \mu_{1}, \ldots\right\}$ is defined by the property

$$
(-1)^{n} \Delta^{n} \mu_{k} \geqslant 0, \quad k, n=0,1, \ldots,
$$

Received 25 November, 1982. 
where

$$
\Delta^{0} \mu_{k}=\mu_{k}, \quad \Delta^{n} \mu_{k}=\Delta^{n-1} \mu_{k+1}-\Delta^{n-1} \mu_{k},
$$

for $k=0,1, \ldots, n=1,2, \ldots$.

This concept is intimately associated with the little moment problem and with Hausdorff summability. A brief survey is found in [11, Chapter III].

F. Hausdorff established (cf. [11, Theorem 4a, p. 108]) the fundamental result for such sequences, as follows.

THeOREM C. A sequence $\left\{\mu_{k}\right\}_{0}^{\infty}$ is completely monotonic if and only if there exists a bounded non-decreasing function $\beta(t), 0 \leqslant t \leqslant 1$, such that

$$
\mu_{k}=\int_{0}^{1} t^{k} d \beta(t), \quad k=0,1, \ldots
$$

Strict inequality prevails throughout (3) for a completely monotonic sequence unless $\mu_{1}=\mu_{2}=\ldots=\mu_{n}=\ldots$, that is, unless all terms except possibly its first are identical [6]. Compare [8] for monotonicity of finite order.

If (1) (respectively (3)) holds for $n=0,1, \ldots, N$, then $f(x)$ (respectively $\left.\left\{\mu_{k}\right\}_{0}^{\infty}\right)$ is said to be monotonic of order $N$. If (3) holds when modified by the deletion of the factor $(-1)^{n}$, then $\left\{\mu_{k}\right\} 0$ is said to be absolutely monotonic, as for functions.

Both types of higher monotonicity can be subsumed into the concept of regular monotonicity introduced by S. N. Bernstein [1, pp. 196-197; 2]. This concept is surveyed in [3] and examined further in [9]. Regularly monotonic functions (respectively sequences) are functions (respectively sequences) whose $n$-th derivative (respectively difference) is of constant sign over the prescribed domain of definition. When this domain is $(a, \infty)$ for functions (respectively the set of all non-negative integers for sequences), F. Neuman and J. Vosmanský [9] have determined all possible permissible successions of signs of the derivatives (respectively differences) of order $n$, for $n=0,1,2, \ldots$.

Many of the results below can be stated in terms of these various types, especially those proved by means of L'Hospital's rule.

A close relationship exists between completely monotonic functions and completely monotonic sequences. For example, as a major step in a proof of Bernstein's Theorem A above, the following result is employed [11, Theorem 11d, p. 158].

THEOREM D. If $f(x)$ is completely monotonic in $[a, \infty)$ and if $\delta$ is any fixed positive number, then the sequence $\{f(a+k \delta)\}_{0}^{\infty}$ is completely monotonic.

2. Composition of completely monotonic functions and sequences

Theorem D can be generalized.

THEOREM 1. If $W(x)$ is completely monotonic on $[a, \infty)$ and if $\left\{\Delta x_{k}\right\}_{0}^{\infty}$ is completely monotonic, with $x_{0} \geqslant a$, then so is the sequence $\left\{W\left(x_{k}\right)_{0}^{\infty_{0}^{\infty}}\right.$.

Proof. From Hausdorff's Theorem C above we note that there exists a bounded 
non-decreasing function $\beta(t), 0 \leqslant t \leqslant 1$, for which

$$
\Delta x_{k}=\int_{0}^{1} t^{k} d \beta(t), \quad k=0,1,2, \ldots
$$

Hence

and so

$$
x_{k}-x_{0}=\sum_{j=0}^{k-1} \Delta x_{j}=\int_{0}^{1} \sum_{j=0}^{k-1} t^{j} d \beta(t)=\int_{0}^{1} \frac{1-t^{k}}{1-t} d \beta(t)
$$

$$
x_{k}=x_{0}+\int_{0}^{1} \frac{1-t^{k}}{1-t} d \beta(t), \quad k=0,1,2, \ldots
$$

Now consider the function

$$
x(k)=x_{0}+\int_{0}^{1} \frac{1-t^{k}}{1-t} d \beta(t)
$$

where $k$ is a continuous variable, $k \geqslant 0$. Then

$$
(-1)^{n} x^{(n+1)}(k)=(-1)^{n} \int_{0}^{1} t^{k} \frac{\log ^{(n+1)} t}{t-1} d \beta(t) \geqslant 0, \quad k>0, n=0,1,2, \ldots,
$$

so that $x^{\prime}(k)$ is a completely monotonic function over $0<k<\infty$.

Hence, $W(x(k))$ is a completely monotonic function over $0<k<\infty$, as may be inferred from Faa di Bruno's formula for the $n$-th derivative of a composite function.

From the definition of $x(k)$ it is obvious that

$$
x(k+\varepsilon)-x(k)=\int_{0}^{1} t^{k} \frac{1-t^{\varepsilon}}{1-t} d \beta(t), \quad \varepsilon>0,
$$

whence

$$
\lim _{\varepsilon \rightarrow 0+}[x(k+\varepsilon)-x(k)]= \begin{cases}0, & k>0, \\ \beta(0+)-\beta(0) \geqslant 0, & k=0 .\end{cases}
$$

Therefore, $x(k+)=x(k)$, for $k>0$, and $x(0+)=x_{0}+\beta(0+)-\beta(0) \geqslant x_{0}$.

Further,

$$
x_{1}=x(1)=x_{0}+\int_{0}^{1} d \beta(t)=x_{0}+\beta(1)-\beta(0) \geqslant x_{0}+\beta(0+)-\beta(0)=x(0+) .
$$

The sequence $\{W(x(k+\varepsilon))\}_{k=0}^{\infty}$ is completely monotonic for each fixed $\varepsilon>0$, 
since the function $W(x(k))$ is completely monotonic for $k>0$. Thus,

$$
(-1)^{n} \Delta^{n} W(x(k+\varepsilon)) \geqslant 0, \quad n, k=0,1,2, \ldots,
$$

and so, letting $\varepsilon \rightarrow 0+$,

$$
(-1)^{n} \Delta^{n} W(x(k+)) \geqslant 0, \quad n, k=0,1,2, \ldots .
$$

That is, the sequence $\left\{W(x(0+)), W\left(x_{1}\right), \ldots, W\left(x_{k}\right), \ldots\right\}$ is completely monotonic, since $x_{0} \leqslant x(0+) \leqslant x_{1}$.

If $x(0+)=x_{1}$, then $\beta(0)=\beta(0+)=\beta(1)$ and so $\beta(x)=\beta(0)$, for $0<x \leqslant 1$. In this case, $\Delta x_{k}=0$, for $k=1,2, \ldots$, and the conclusion of Theorem 1 holds trivially; the sequence $\left\{W\left(x_{k}\right)_{0}^{\infty}\right.$ is simply a sequence of constants.

In any case, $x(0+) \geqslant x_{0}$, so that

$$
W\left(x_{0}\right) \geqslant W(x(0+)) .
$$

Thus, from (7), the sequence $\left\{W\left(x_{k}\right)_{0}^{\infty \infty}\right.$ is completely monotonic, as the theorem asserts, since increasing the first term in a completely monotonic sequence does not affect the property of complete monotonicity.

The theorem is proved.

REMARK 1. If $x_{0}>a$, then it suffices to assume that $W(x)$ is completely monotonic over $(a, \infty)$, and the foregoing proof can be simplified a bit.

Remark 2. As a preliminary to Corollary 1 we require an analogue of an easy modification of Theorem $2 b$ of $[11$, p. 145]. This is Lemma 1 below, the proof of which is accomplished by the use, again, of the interplay between completely monotonic functions and sequences, an interplay which makes the lemma a consequence as well as an analogue of that theorem.

LemMA 1. If $f_{1}(x)$ is absolutely monotonic in $[0, \infty)$, and if $\left\{\mu_{k}\right\}_{0}^{\infty}$ is completely monotonic, then $\left\{f_{1}\left(\mu_{k}\right)\right\}$ is completely monotonic. In particular, if $\left\{\log v_{k}\right\}$ is completely monotonic (so that $v_{k} \geqslant 1$ ), then so also is $\left\{v_{k}^{\alpha}\right\} 0$ for each $\alpha>0$.

Proof. First, assume that $\left\{\mu_{k}\right\}_{0}^{\infty}$ is minimal. Then, by [11, Theorem 14b, p. 164], there exists a function $f_{2}(x)$, completely monotonic over $[0, \infty)$, such that

$$
f_{2}(k)=\mu_{k}, \quad k=0,1,2, \ldots
$$

From Theorem $2 \mathrm{~b}$ of $\left[11\right.$, p. 145], modified trivially, it follows that $f_{1}\left(f_{2}(x)\right)$ is completely monotonic over $[0, \infty)$. Hence, the sequence $\left\{f_{1}\left(f_{2}(k)\right)\right\}_{0}^{\infty} \equiv\left\{f_{1}\left(\mu_{k}\right)\right\}_{0}^{\infty}$ is completely monotonic, from [11, Theorem 11d, p. 158]; this proves the first part of the lemma in this case.

If $\left\{\mu_{k}\right\}_{0}^{\infty}$ is not minimal, it can be made so by reducing the single term $\mu_{0}$ appropriately, say to $\mu_{0}^{*}$. The preceding argument establishes that the sequence $\left\{f_{1}\left(\mu_{0}^{*}\right), f_{1}\left(\mu_{1}\right), \ldots\right\}$ is completely monotonic. Hence so also is $\left\{f_{1}\left(\mu_{k}\right)_{\}_{0}}^{\}_{0}}\right.$, since $f_{1}(x)$ increases as $x$ increases; the sequence obtained by increasing the first term of a completely monotonic sequence is completely monotonic. This proves the first part of the lemma. 
To establish the remaining part, simply take $f_{1}(x)=e^{\alpha x}, \mu_{k}=\log v_{k}$.

REMARK 3. The preceding remarks permit the assertion of a consequence of Theorem 1, modified as indicated in Remark 1.

COROLlaRY 1. If $\left\{\Delta x_{k}\right\}_{0}^{\infty}$ is completely monotonic, then so is the sequence (i) $\left\{\exp \left(-\lambda x_{k}\right)\right\}_{0}^{\infty}$ when $x_{0} \geqslant 0$; and when $x_{0}>0$, so also are the sequences (ii) $\left\{x_{k}^{-\lambda}\right\}_{0}^{\infty}$, (iii) $\left\{\log \left(x_{k+1} / x_{k}\right)\right\}_{0}^{\infty}$, and (iv) $\left\{\left(x_{k+1} / x_{k}\right)^{\lambda}\right\}_{0}^{\infty}$, for each fixed $\lambda>0$.

Proof. For (i) and (ii) it suffices to take $W(x)=e^{-\lambda x}$ and $W(x)=x^{-\lambda}$, respectively, in Theorem 1 . From (ii) the complete monotonicity of (iii) can be inferred via L'Hospital's rule, since for $\lambda>0$,

$$
\begin{aligned}
0 \leqslant & \lambda^{-1}(-1)^{n+1} \Delta^{n+1} x_{k}^{-i}=(-1)^{n+1} \Delta^{n}\left\{\frac{x_{k+1}^{-\lambda}-x_{k}^{-\lambda}}{\lambda}\right\} \\
& \rightarrow(-1)^{n+1} \Delta^{n}\left\{-\log \left(x_{k+1}\right)+\log x_{k}\right\}=(-1)^{n} \Delta^{n}\left\{\log \left(x_{k+1} / x_{k}\right)\right\},
\end{aligned}
$$

as $\lambda \rightarrow 0+$, for $k, n=0,1, \ldots$. This settles case (iii).

The special case when $\lambda=1$ of (iv) can be handled especially simply, since

$$
x_{k+1} / x_{k}=\left(\Delta x_{k}\right)\left(x_{k}^{-1}\right)+1 .
$$

This establishes the complete monotonicity of the sequence $\left\{x_{k+1} / x_{k}\right\}_{0}^{\infty}$, since its $k$-th term is the product and sum of the $k$-th terms of completely monotonic sequences.

The general case when $\lambda>0$ of (iv) follows from (iii) and the final sentence of Lemma 1 on putting

$$
v_{k}=x_{k+1} / x_{k}, \quad k=0,1,2, \ldots .
$$

This completes the proof of Corollary 1.

Remark 4. To the second part of Lemma 1 there is a valid converse, as follows.

LEMMA 2. If $\left\{v_{k}^{\lambda\}_{0}^{\infty}}\right.$ is completely monotonic for each $\lambda>0$, then so is $\left\{\log v_{k}\right\}_{0}^{\infty}$, provided that $v_{k} \geqslant 1$, for $k=0,1,2, \ldots$.

Proof. For $v_{k}>0$,

$$
0 \leqslant \lambda^{-1}(-1)^{n} \Delta^{n} v_{k}^{\lambda}=(-1)^{n} \Delta^{n}\left\{\frac{v_{k}^{\dot{\lambda}}-1}{\lambda}\right\} \rightarrow(-1)^{n} \Delta^{n} \log v_{k},
$$

as $\lambda \rightarrow 0+$ for $n=1,2, \ldots, k=0,1,2, \ldots$. Moreover (since $v_{k} \geqslant 1$ ),

$$
(-1)^{0} \Delta^{0} \log v_{k}=\log v_{k} \geqslant 0, \quad k=0,1,2, \ldots ;
$$

this completes the proof.

Remark 5. A similar argument shows the following. 
LEMMA 3. If $\left\{\mu_{k}^{i}\right\}_{0}^{\infty}$ is completely monotonic for each $\lambda>0,0<\mu_{k}$, $k=0,1,2, \ldots$, then $\left\{\log \left(\mu_{k} / \mu_{k+1}\right)\right\}_{0}^{\infty}$ is completely monotonic.

Proof. For $\lambda>0$, and $n=1,2, \ldots, k=0,1,2, \ldots$,

$$
\begin{aligned}
0 \leqslant \lambda^{-1}(-1)^{n} \Delta^{n} \mu_{k}^{\lambda} & =(-1)^{n} \Delta^{n-1}\left\{\frac{\mu_{k+1}^{\lambda}-\mu_{k}^{\lambda}}{\lambda}\right\} \\
& \rightarrow(-1)^{n} \Delta^{n-1}\left\{\log \mu_{k+1}-\log \mu_{k}\right\}=(-1)^{n-1} \Delta^{n-1} \log \left(\mu_{k} / \mu_{k+1}\right),
\end{aligned}
$$

as $\lambda \rightarrow 0+$.

From this result, it follows that the complete monotonicity result stated as [7, (iv), p. 1255] is more precise than indicated previously. The assertion in question states that the sequence $\left\{\exp \left(-\lambda c_{v k}\right)\right\}_{2}^{\infty}$ is completely monotonic for each $\lambda>0$, where $c_{v k}$ is the $k$-th positive zero of the general real Bessel function $\mathscr{C}_{v}(x)$, when $|v| \geqslant \frac{1}{2}$. In view of the new result, it follows that whenever $\left.\exp \left(-\lambda c_{v k}\right)\right\}_{2}^{\infty}$ is completely monotonic, the sequence $\left\{\Delta c_{v k}\right\}_{2}^{\infty}$ must also be completely monotonic. But this is the case when and only when $|v| \geqslant \frac{1}{2}$. Thus the earlier result does not hold for a larger range of $v$ than the one already stated there.

Remark 6. Lemma 3 and Corollary 1(i) are converse to one another. Together they constitute an analogue for completely monotonic sequences of Schoenberg's Theorem 9 [10, p. 835] for completely monotonic functions.

To parallel his phrasing, the results may be combined as follows.

The sequence $\left\{\mu_{k}^{i}\right\}_{0}^{\infty}, \mu_{0}=1, \mu_{k}>0, k=1,2, \ldots$, is completely monotonic for all $\lambda>0$ if and only if $\mu_{k}=\exp \left(-v_{k}\right)$ with $\left\{\Delta v_{k}\right\}_{0}^{\infty}$ completely monotonic and $v_{0}=0$.

REMARK 7. Theorem 1 generalizes not only Theorem D, but also the case when $N=\infty$ of [7, Corollary 3.1], itself a substantial generalization of Theorem $\mathrm{D}$. The previous extension, however, assumes that $x_{0}, x_{1}, \ldots, x_{k}, \ldots$ are the zeros of a solution of a certain type of differential equation, which is the case when $x_{k}=a+k \delta$.

\section{Another composition of completely monotonic functions and sequences}

Still another result of [7], namely the case when $N=\infty$ of Corollary 3.2, can be freed of dependence on differential equations and thus generalized. The extension is the following.

THEOREM 2. Suppose that $V^{\prime}(x)$ is completely monotonic over $(0, \infty)$, that $x_{k}$ is in the domain of $V(x)$ and that $\left\{\Delta x_{k}\right\}_{0}^{\infty}$ is completely monotonic. Then the sequence $\left\{\Delta V\left(x_{k}\right)\right\}_{0}^{\infty}$ is also completely monotonic.

Proof. As in the proof of Theorem 1, the sequence $\left\{x_{k}\right\}$ can be extended to a function $x(k)$ such that $x^{\prime}(k)$ is a completely monotonic function of the continuous variable $k$, for $k>0$. Similarly it follows again from Faa di Bruno's formula that $V^{\prime}(x(k))$ is also a completely monotonic function of $k$. Hence the same is true of the 
product $x^{\prime}(k) V^{\prime}(x(k))$ and we may write

$$
\frac{d V(x(k))}{d k}=x^{\prime}(k) V^{\prime}(x(k))=\int_{0}^{1} t^{k} d \gamma(t),
$$

where $d \gamma(t)$ is a positive measure obtained from Bernstein's Theorem B on replacing $e^{-t}$ by $t$.

Integration from $k$ to $k+1$ gives

$$
V(x(k+1))-V(x(k))=\int_{0}^{1} \frac{t-1}{\log t} t^{k} d \gamma(t),
$$

since $\int_{k}^{k+1} t^{r} d r=\frac{t-1}{\log t} t^{k}$

Now, $(t-1) / \log t$ is non-negative and continuous in $[0,1]$, which shows that $V(x(k+1))-V(x(k))$ is completely monotonic, so that the sequence $\left\{\Delta V\left(x_{k}\right)\right\}$ is also completely monotonic, as claimed.

Remark. Theorems 1 and 2 show that Corollaries 3.1 and 3.2 of [7] can be freed from their dependence on differential equations when $N=\infty$ and are general properties of completely monotonic functions and sequences. However, a similar result of [7], namely Theorem 3.2, cannot be extended in this way. To see this, we note that there exist sequences $\left\{x_{k}\right\},\left\{t_{k}\right\}$, with $\left\{\Delta x_{k}\right\},\left\{\Delta t_{k}\right\}$ both completely monotonic, with

$$
x_{k}>t_{k}>x_{k-1}>0, \quad x_{0}>t_{0}, \quad k=1,2, \ldots,
$$

and such that the sequence $\left\{w\left(x_{k}\right)-w\left(t_{k}\right)\right\}_{0}^{\infty}$ need not be monotonic, much less completely monotonic, although $W(x)=w^{\prime}(x)$ is a completely monotonic function.

It suffices to let

$$
w(x)=x, \quad x_{k}=k+\frac{1}{k+1}, \quad t_{k}=k+\frac{a}{(k+1)^{2}},
$$

with $a$ any fixed value, $\frac{2}{3}<a<1$.

For $\left\{x_{k}\right\},\left\{t_{k}\right\}$ the zeros of solutions of a certain type of Sturm-Liouville differential equation the sequence $\left\{w\left(x_{k}\right)-w\left(t_{k}\right)\right\}_{0}^{\infty}$ would be completely monotonic, according to Theorem 3.2 of [7], whenever $w^{\prime}(x)$ is completely monotonic.

\section{A partial converse to Theorem 1}

A partial converse to Theorem 1 holds. It is analogous to one of the two directions of a necessary and sufficient condition established by I. J. Schoenberg [10, Theorem 8, p. 833] for the superposition of one completely monotonic function upon the derivative of another. Theorem 1 is the corresponding analogue to the opposite direction of Schoenberg's theorem. The formulation below imposes a milder 
hypothesis, restricting itself to the family generated by a single positive, decreasing, differentiable function, rather than covering the totality of all completely monotonic functions. Examples of a suitable $W(x)$ are $e^{-x}$ or $1 /(x+1)$.

Theorem 3. Let $W(x)>0$, for $0 \leqslant x<\infty$, with $W^{\prime}(x)<0$, for $0<x<\infty$, and suppose that $W^{\prime}(0+)$ exists as a finite value. If $\left\{W\left(\lambda x_{k}\right)\right\}_{0}^{\infty}$ is completely monotonic for all small $\lambda>0$, and if $x_{0} \geqslant 0$, then $\left\{\Delta x_{k}\right\}$ is completely monotonic.

Proof. Obviously, $-\infty<W^{\prime}(0+) \leqslant 0$. For $\lambda>0$ and $n=1,2, \ldots$, we have, using L'Hospital's rule,

$0 \leqslant(-1)^{n} \Delta^{n}\left\{\lambda^{-1} W\left(\lambda x_{k}\right)\right\}=(-1)^{n} \Delta^{n}\left\{\frac{W\left(\lambda x_{k}\right)-W(0)}{\lambda}\right\}$

Hence,

$$
\rightarrow(-1)^{n} W^{\prime}(0+) \Delta^{n} x_{k}, \quad \text { as } \lambda \rightarrow 0+.
$$

$$
(-1)^{n-1} \Delta^{n-1}\left(\Delta x_{k}\right) \geqslant 0, \quad n=1,2,3, \ldots, \quad k=0,1,2, \ldots,
$$

and the proof is complete.

Remark 1. If, instead of supposing that $W^{\prime}(x)<0$, it is assumed that $W^{\prime}(x)>0$, as, say, when $W(x)=e^{x}$, then the theorem holds when absolute monotonicity replaces complete monotonicity in both hypothesis and conclusion. In both cases, the theorem would remain valid in finite form. With the appropriate hypothesis assumed for $n=0,1, \ldots, N$, the corresponding conclusion would hold for $n=0,1, \ldots, N-1$.

It is easy to formulate the corresponding statement for regular monotonicity. The same proof works, once $(-1)^{n}$ is replaced by $\varepsilon_{n}$, where $\varepsilon_{n}$ is \pm 1 as appropriate.

These comments apply, mutatis mutandis, to Corollary 1 ((ii) implies (iii)), Lemma 2 and Lemma 3, above.

Remark 2. A full converse to Theorem 1 does not hold. Below we shall show this by establishing Theorem 4 .

\section{Some counterexamples}

We have already mentioned, in the remark following Theorem 2, a counterexample to a possible extension of a result having an origin similar to Theorems 1 and 2.

(1) Next we show that the Schoenberg result mentioned in $\$ 4$ cannot be improved by weakening the hypotheses so as to require only the complete monotonicity of $f(\phi(t))$ for a single completely monotonic $f(t)$. The result follows.

THEOREM 4. For each function $f(t)$ completely monotonic over $[0, \infty)$ there exists a function $\phi(t)$ such that $\phi(0)=0, f(\phi(t))$ is completely monotonic over $[0, \infty)$ and $\phi^{\prime}(t)$ is not completely monotonic on $(0, \infty)$. 
Proof. For $f(t)$ constant the conclusion is obvious, and so we consider hereafter the non-constant case. Obviously, $f(\infty)$ exists and is non-negative. By subtracting this value, we may as well assume that $f(\infty)=0$. Further, $f(0)>0$, since any complete monotonic function is non-negative and non-increasing. Hence we may assume without loss of generality that $f(0)=1$.

This done, we note also that $f(t)$ is strictly decreasing, since $\left(1^{\prime}\right)$ holds.

Next, given $f(t)$, we construct $\phi(t)$ with $\phi(0)=0$ such that

$$
f(\phi(x))=e^{-x} .
$$

Then $f^{\prime}(\phi(x)) \phi^{\prime}(x)=-e^{-x}$ and

$$
\begin{aligned}
\phi^{\prime \prime}(x) & =\frac{e^{-x}-f^{\prime \prime}(\phi(x))\left[\phi^{\prime}(x)\right]^{2}}{f^{\prime}(\phi(x))}=\frac{e^{-x}-f^{\prime \prime}(\phi(x))\left[f^{\prime}(\phi(x))\right]^{-2} e^{-2 x}}{f^{\prime}(\phi(x))} . \\
e^{x} \phi^{\prime \prime}(x) & =\frac{\left[f^{\prime}(\phi(x))\right]^{2}-f^{\prime \prime}(\phi(x)) e^{-x}}{\left[f^{\prime}(\phi(x))\right]^{3}} \\
& =\frac{\left[f^{\prime}(\phi(x))\right]^{2}-[f(\phi(x))]\left[f^{\prime \prime}(\phi(x))\right]}{\left[f^{\prime}(\phi(x))\right]^{3}}, \quad x>0,
\end{aligned}
$$

since $f(\phi(x))=e^{-x}$.

The last numerator is non-positive [11, comment after proof of Theorem 16, p. 167], while the denominator is negative from $\left(1^{\prime}\right)$. Hence

$$
\phi^{\prime \prime}(x) \geqslant 0, \quad x>0 .
$$

If $\phi^{\prime \prime}(x)>0$ for some $x$, then $\phi^{\prime}(x)$ is clearly not decreasing for all $x>0$, as would be required for complete monotonicity, and the proof would be complete.

If, on the other hand, $\phi^{\prime \prime}(x)=0$ for all $x>0$, then $\phi(x)=c x$ for all $x$, where $c>0$, since $\phi(0)=0$ and $\phi^{\prime}(x)>0$.

In this case, we have $f(c x)=e^{-x}$, which can be written

$$
f(x)=e^{-\alpha x}, \quad \text { where } 0<\alpha=\frac{1}{c} .
$$

That is, the theorem has been established for all completely monotonic functions over $[0, \infty)$ except when $f(x)=e^{-\alpha x}, \alpha>0$.

To prove it for this case, an ad hoc construction is available. Define

$$
\phi(x)=-\frac{1}{\alpha} \log \frac{1+2 e^{-x}}{3},
$$

so that $\phi(0)=0$ and $f(\phi(x))=\frac{2}{3} e^{-x}+\frac{1}{3}$, which is completely monotonic over $[0, \infty)$.

Now,

$$
\phi^{\prime}(x)=\frac{1}{\alpha} \frac{2}{e^{x}+2}
$$


and, with $y=e^{x}$, so that $y \geqslant 1$,

$$
\phi^{\prime \prime}(x)=-\frac{1}{\alpha} \frac{2}{y+4+(4 / y)} .
$$

The denominator decreases for $1 \leqslant y \leqslant 2$ (that is, $0 \leqslant x<\log 2$ ) and increases for $y>2$ (that is, $x>\log 2$ ), since its derivative with respect to $y$ is $1-4 y^{-2}$. Thus, $\phi^{\prime \prime \prime}(x)$ is not of constant sign for $0<x<\infty$ and $\phi^{\prime}(x)$ cannot be convex, much less completely monotonic on $(0, \infty)$.

The proof is now complete.

(2) A function completely monotonic in $[0, \infty)$, although necessarily analytic in $(0, \infty)$, need not be analytic at the end-point $x=0$, even if all derivatives $f^{(k)}(0+)$ are finite, for $k=0,1,2, \ldots$. An example of such a function is

$$
f(x)=\int_{0}^{\infty} e^{-x t} d \alpha(t), \quad \alpha(t)=1-\exp \left(-t^{1 / 2}\right) .
$$

The abscissa of convergence of this transform is $x=0$ [11, Theorem $2.4 \mathrm{~d}, \mathrm{p} .43]$. Hence $x=0$ is a singular point of $f(x)$, from the Laplace-Stieltjes analogue [11, Theorem 5b, p. 58] of the Pringsheim-Vivanti theorem for power series, since $\alpha(t)$ is monotonic.

On the other hand, $f(0+)$ exists, since $\alpha(t)$ is bounded, from Bernstein's Theorem A. To examine the derivatives at the origin, we write first

$$
(-1)^{k} f^{(k)}(x)=\int_{0}^{\infty} e^{-x t} t^{k} d \alpha(t) \equiv \int_{0}^{\infty} e^{-x t} d \alpha_{k}(t)
$$

where

$$
\alpha_{k}(t)= \begin{cases}\int_{0}^{1} y^{k} d \alpha(y)=\int_{0}^{1} k y^{k-1}[\alpha(t)-\alpha(y)] d y, & k=1,2, \ldots, \\ \alpha(t), & k=0 .\end{cases}
$$

Appealing again to Bernstein's Theorem A, since $(-1)^{k} f^{(k)}(x)$ is completely monotonic in $(0, \infty)$ for each $k=0,1,2, \ldots$, we note that $f^{(k)}(0+)$ exists if and only if $\alpha_{k}(\infty)<\infty$. Here

$$
\alpha_{k}(t)=\int_{0}^{t} y^{k} d \alpha(y)=\frac{1}{2} \int_{0}^{1} y^{k-(1 / 2)} \exp \left(-y^{1 / 2}\right) d y=\int_{0}^{k 1} r^{2 k} e^{-r} d r,
$$

and so

$$
\alpha_{k}(\infty)=\Gamma(2 k+1), \quad k=0,1,2, \ldots,
$$

a finite value.

This establishes the assertion. 
The function $f(x)$ can be expressed in terms of tabulated functions [5, $3.322(2)$, p. $307,8.250(1)$, p. 930$]$

$$
f(x)=\frac{1}{2}(\pi / x)^{1 / 2}[\exp \{1 /(4 x)\}]\left[1-\Phi\left(\frac{1}{2} x^{-1 / 2}\right)\right],
$$

where $\Phi(t)$ is the probability integral

$$
\Phi(t)=2 \pi^{-1 / 2} \int_{0}^{t} \exp \left(-r^{2}\right) d r .
$$

(3) For a function $f(x)$ completely monotonic over $[0, \infty)$, the derivatives $f^{(n)}(0+)$ can exist for $n=0,1, \ldots, N-1$, while $f^{(N)}(0+)$ fails to exist. We illustrate this solely for the case when $N=1$. In this instance, define

$$
\alpha(t)=1-(1+t)^{-1},
$$

a bounded increasing function, so that $f(0+)$ exists and the function $-f^{\prime}(x)$ is completely monotonic over $(0, \infty)$. But

$$
-f^{\prime}(x)=\int_{0}^{\infty} e^{-x t} t(1+t)^{-2} d t=\int_{0}^{\infty} e^{-x t} d \beta(t)
$$

with $\beta(t)=\log (1+t)+(1+t)^{-1}$, an unbounded function as $t \rightarrow \infty$, so that $f^{\prime}(0+)$ does not exist.

In other words, $f(x)$ is completely monotonic over $[0, \infty)$ while $-f^{\prime}(x)$ is completely monotonic only over $(0, \infty)$.

Another, analytically simpler, example is obtained by making an obvious modification of a function exhibited in [11, Example 7, p. 145] for a similar purpose. Widder's modified example is arcsin $e^{-x}$. In our case, $f(x)$ can again be found in tables $[5,3.353(3)$, p. 311]

$$
f(x)=1-x e^{x} \int_{x}^{\infty} t^{-1} e^{-t} d t, \quad x>0, f(0)=1 .
$$

\section{On the superposition of completely and absolutely monotonic functions}

Here we provide something of an analogue to the Schoenberg result cited in $\$ 4$. There the outer function ranged over a family of functions while the inner function remained fixed. The reverse is the case below.

THEOREM 5. If $\phi(f(x))$ is completely monotonic over $(0, \infty)$ for all functions $f(x)$ which are completely monotonic over $(0, \infty)$, then $\phi(x)$ is absolutely monotonic over $(0, \infty)$.

Proof. First we show that $\phi(z)$ is an entire function, and then that its power 
series expansion has exclusively non-negative coefficients. This will prove the theorem, in view of the uniqueness theorem for Laplace-Stieltjes transforms [11, Theorem 6.3, p. 63].

The function $c+\rho e^{-x}$ is completely monotonic for $\rho>0$, and any constant $c \geqslant 0$, so that $\phi\left(c+\rho e^{-x}\right)$ is also completely monotonic. Hence, $\phi\left(c+\rho e^{-x}\right)$ is analytic for $\mathscr{R} x>0\left[11\right.$, p. 146]. But $c+\rho e^{-x}$ fills the punctured disk $0<|z-c|<\rho$ as $x$ fills $\mathscr{R} x>0$. Thus, $\phi(z)$ is analytic throughout $0<|z-c|<\rho$ for any positive $\rho$. Taking any two values of $c$, say $c=0$ and $c=1$, we see that $\phi(z)$ is entire, since $\rho$ is arbitrary.

Now let $\phi(z)=\sum_{k=0}^{\infty} a_{k} z^{k}$. By hypothesis

$$
\phi\left(e^{-x}\right)=\sum_{k=0}^{\infty} a_{k} e^{-k x}
$$

is completely monotonic, since $e^{-x}$ is. But (11) is the (unique) Laplace-Stieltjes transform of masses $\left\{a_{k}\right\}_{0}^{\infty}$ placed at $k$ and so each $a_{k} \geqslant 0, k=0,1,2, \ldots$, as required.

Remark 1. The converse of Theorem 5 also holds (cf. [11, Theorem 2b, p. 145]).

Remark 2. Theorem 5 has the following special case.

Corollary 2. $[f(x)]^{i}, \lambda \geqslant 0$, cannot be completely monotonic for all completely monotonic functions $f(x)$ unless $\lambda=0,1,2, \ldots$.

\section{Remarks on Schoenberg's Theorems 8 and 9}

These Schoenberg theorems deal solely with the properties of completely monotonic functions, rather than the interplay between such functions and metric spaces. Schoenberg's proofs utilize the interplay, but this is not necessary. Those results can be verified in more or less the same spirit as the proofs of the theorems in this paper. Where limits are taken, however, complications arise now, since this requires interchange of limit and derivative (or evasion of this interchange). In results on sequences, only the interchange of limit and finite sums arose.

One direction of Schoenberg's Theorem $8 \quad[10$, p. 833] is really contained in the same direction of his Theorem 9 [10, p. 835]. (The converse direction of his Theorems 8 and 9 can be established by Faa di Bruno's formula for the higher derivatives of composite functions.) The Schoenberg result for which we offer an alternative proof follows.

THEOREM E. If $\exp \{-\lambda \phi(t)\}$ is completely monotonic over $[0, \infty)$ for each $\lambda>0$, where $\phi(t) \geqslant 0, \phi(0)=\phi(0+)=0$, then $\phi^{\prime}(t)$ is completely monotonic over $(0, \infty)$.

Proof. Clearly, all derivatives of

$$
\phi(t)=-\log \exp \{-\phi(t)\}
$$

exist over $(0, \infty)$. Moreover, $\phi^{\prime}(t) \geqslant 0$, since $e^{-x}$ is a decreasing function of $x$ and $\exp \{-\phi(t)\}$ is non-increasing, being completely monotonic. 
Further, the hypothesis implies that, for $n=1,2, \ldots$, and $\lambda>0$,

$0 \leqslant(-1)^{n} \frac{d^{n} \exp \{-\lambda \phi(t)\}}{\lambda d t^{n}}=(-1)^{n-1} \frac{d^{n-1}\left[\phi^{\prime}(t) \exp \{-\lambda \phi(t)\}\right]}{d t^{n-1}}, \quad 0<t<\infty$.

Hence, the function $\phi^{\prime}(t) \exp \{-\lambda \phi(t)\}$ is completely monotonic for $0<t<\infty$ and $\lambda>0$. As the pointwise limit of completely monotonic functions, $\phi^{\prime}(t)$ is also completely monotonic over $(0, \infty)$.

Alternatively, the proof may be concluded by applying Leibniz's rule [5, p. 19] for the $n$-th derivative of a product. This implies that

$$
\frac{d^{n-1}\left[\phi^{\prime}(t) \exp \{-\lambda \phi(t)\}\right]}{d t^{n-1}}=[\exp \{-\lambda \phi(t)\}] \phi^{(n)}(t)+\lambda[\ldots]
$$

where [...], which can be given explicitly, approaches a finite value as $\lambda \rightarrow 0+$. Thus,

$$
\lim _{i \rightarrow 0+} \frac{d^{n-1}\left\{\phi^{\prime}(t) \exp (-\lambda \phi(t))\right\}}{d t^{n-1}}=\phi^{(n)}(t), \quad 0<t<\infty, n=1,2, \ldots
$$

Therefore,

$$
0 \leqslant(-1)^{n-1} \phi^{(n)}(t), \quad n=1,2, \ldots, \quad 0<t<\infty,
$$

so that $\phi^{\prime}(t)$ is completely monotonic over $(0, \infty)$, as claimed.

The converse direction of Theorems 8 and 9 [10, pp. 833-835] can be formulated as follows.

THEOREM $\mathrm{E}^{\prime}$. If $\phi(t)$ is a continuous, non-negative function with $\phi(0+)=0$ and $\phi^{\prime}(t)$ completely monotonic over $(0, \infty)$, then $f(\phi(t))$ is completely monotonic on $[0, \infty)$ whenever $f(t)$ is completely monotonic over $[0, \infty)$.

A proof can be achieved again by Leibniz's rule [5, p. 19] on writing

$$
(-1)^{n} \frac{d^{n} f(\phi(t))}{d t^{n}}=(-1)^{n} \frac{d^{n-1}}{d t^{n-1}}\left\{f^{\prime}(\phi(t)) \phi^{\prime}(t)\right\} .
$$

Details are omitted.

Remark 1. The foregoing proofs are applicable to monotonicity of finite order, unlike those in [10]. Assuming monotonicity of order $N$ in the hypotheses would lead to monotonicity of order $N-1$ in the conclusions. Moreover, this method of proof permits the establishment of analogous theorems for absolute and regular monotonicity.

Remark 2. In Theorem E, the function $e^{-x}$ may be replaced by any function $W(x)$ completely monotonic over $[0, \infty)$, provided that $-W^{\prime}(x)$ is completely monotonic over $[0, \infty)$ as well, that is, provided that $W^{\prime}(0+)$ exists as a finite value. 


\section{Some comments on monotonicity of finite order}

We have pointed out that various of the foregoing proofs hold for monotonicity of finite order (those of Theorems 1 and 2 are among the exceptions). Typically, monotonicity of order $N$ in the hypotheses would imply monotonicity of order $N-1$ in a number of the conclusions.

Here we present some miscellaneous results somewhat related to this.

THEOREM 6. If $f(t)$ and $g(t)$ are convex and non-negative, and if $k \geqslant 1$, then $\left\{[f(t)]^{k}+[g(t)]^{k}\right\}^{1 / k}$ is also convex.

Proof. The function $g(u, v)=\left(u^{k}+v^{k}\right)^{1 / k}$ is an increasing and convex function of the vector $(u, v)$, from the triangle inequality for the $k$-norm. Further, the composition of an increasing convex function with a vector whose components are both convex is clearly convex, directly from the definition, and so the theorem is proved.

We shall make use of the following consequence.

Corollary 3. For $k \geqslant 1$, any convex combination of functions $[f(t)]^{k}$, where each $f(t)$ is convex and non-negative, is itself of the form $[F(t)]^{k}$, where $F(t)$ is convex.

This in turn yields the following result.

THEOREM 7. If $f(t)$ is monotonic of order $N$, for $N \geqslant 2$, then $[f(t)]^{1 /(N-1)}$ is convex. In particular, if $f(t)$ is monotonic of order 3 , then $[f(t)]^{1 / 2}$ is convex.

Proof. For functions monotonic of order $N$, the extreme points are the functions $(1-a x)_{+}^{N-1}$, where, as usual, $(t)_{+}=t$ when $t \geqslant 0$ and $(t)_{+}=0$ when $t<0$. This is shown in [12]. Each of these functions and $(1-a x)_{+}$are convex and so our assertion follows from the foregoing corollary.

The special case specified will be of use in that part of the proof of the next theorem which is given in detail.

THEOREM 8. If $f(t)$ is monotonic of order $N$, and $\lambda>1$, then $[f(t)]^{\dot{\lambda}}$ is also monotonic of order $N$ provided that $N=1,2,3,4$. The result is false for $N=5$.

Proof. The assertion is trivial when $N=1$ and straightforward when $N=2$. We give the details for $N=3$, but omit the far more complicated calculations for $N=4$. A counter-example establishes the assertion for $N=5$.

First, let $N=3$. Here

$$
\left(f^{\lambda}\right)^{\prime \prime \prime}=\lambda f^{\lambda-3}\left[f^{\prime \prime \prime} f^{2}+3(\lambda-1) f f^{\prime} f^{\prime \prime}+(\lambda-1)(\lambda-2)\left(f^{\prime}\right)^{3}\right] .
$$

Our assertion amounts to showing that this is non-positive. To do so, it will clearly suffice to establish that

$$
3 f f^{\prime} f^{\prime \prime}+(\lambda-2)\left(f^{\prime}\right)^{3}=f^{\prime}\left[3 f f^{\prime \prime}+(\lambda-2)\left(f^{\prime}\right)^{2}\right] \leqslant 0,
$$

since $\lambda>1$ and $f^{\prime \prime \prime}<0$. But $f^{\prime} \leqslant 0$, making this the same as

$$
3 f f^{\prime \prime}+(\lambda-2)\left(f^{\prime}\right)^{2} \geqslant 0 .
$$


The left side increases with $\lambda$ and so it is enough to consider the case when $\lambda=1$, that is, to prove that $3 f f^{\prime \prime} \geqslant\left(f^{\prime}\right)^{2}$. But, in fact, $2 f f^{\prime \prime} \geqslant\left(f^{\prime}\right)^{2}$, since $f^{1 / 2}$ has been shown to be convex by Theorem 7 .

As mentioned, we do not provide the tedious calculations for the case when $N=4$.

An example shows that monotonicity of order 5 for $f(t)$ does not imply monotonicity of order 5 for $[f(t)]^{\lambda}$ for all $\lambda>1$ : take

$$
f(t)=1+2(1-t)_{+}^{4} .
$$

It can be shown that $F^{(5)}(0)>0$, where $F(t)=[f(t)]^{3 / 2}$, thus establishing our claim.

Finally, we record the following without proof. It should be compared with Corollary 2.

THEOREM 9. If $f(t)$ is completely monotonic, and if $\lambda>1$, then $[f(t)]^{\lambda}$ is monotonic of order 5 .

Acknowledgements. The Natural Sciences and Engineering Research Council of Canada provided partial support for this study. We are grateful also to Professor Martin Muldoon for calling [9] to our attention.

\section{References}

1. S. N. BERNSTEIN, Leçons sur les propriétés extrémales et la meilleure approximation des fonctions analytiques d'une variable réelle (Gauthier-Villars, Paris, 1926).

2. S. N. BernStein, 'Sur les fonctions régulièrement monotones', Atti Congresso Int. Mat. Bologna, 2 (1930), 267-275.

3. R. P. BOAS, JR., 'Signs of derivatives and analytic behavior', Amer. Math. Monthly, 78 (1971), 10851093.

4. J. Dubourdieu, 'Sur un théorème de M. S. Bernstein relatif à la transformation de Laplace-Stieltjes', Compositio Math., 7 (1939), 96-111.

5. I. S. GRADSHTEYN and I. M. RYZHIK, Tables of integrals, series and products, Fourth edition, English translation (prepared by Yu. V. Geronimus and M. Yu. Tseytlin, Academic Press, New York, 1965).

6. L. LORCH and L. MOSER, 'A remark on completely monotonic sequences, with an application to summability', Canad. Math. Bull., 6 (1963), 171-173.

7. L. LORCH, M. E. MULdOON and P. SzEGo, 'Higher monotonicity properties of certain SturmLiouville functions, III', Canad. J. Math., 22 (1970), 1238-1265.

8. M. E. Muldoon, 'Elementary remarks on multiply monotonic functions and sequences', Canad. Math. Bull., 14 (1971), 69-72.

9. F. Neuman and J. VoSMANSKÝ, 'On functions (sequences) whose derivatives (differences) do not change signs', Akad. Nauk. Azerbaǐdžan. SSR Dokl., 34 (1978), 8-12.

10. I. J. SCHOENBERG, 'Metric spaces and completely monotone functions', Ann. of Math., 39 (1938), 811841 .

11. D. V. WIDder, The Laplace transform (Princeton University Press, Princeton, 1941).

12. R. E. Williamson, 'Multiply monotone functions and their Laplace transforms', Duke Math. J., 23 (1956), 189-207.

\section{Department of Mathematics, York University, Downsview, Ontario M3J 1P3, Canada.}

\author{
Department of Mathematics, \\ Temple University, \\ Philadelphia, \\ Pennsylvania 19122, \\ U.S.A.
}

